\title{
Experimental production of permanent heart block (total or bundle branch block) without circulatory arrest or extracorporeal circulation
}

\author{
J . W I E B E R D I N K
}

\author{
From the Department of Cardiovascular Surgery, University Hospital, Utrecht, The Netherlands
}

The experimental production of heart block is a tool with which to study cardiac physiology and artificial pacemaking. Acute as well as chronic experiments usually demand stable blocks, produced with minimal injury to the heart. As the production of a block is merely an introduction to the factual experiment, its operative mortality ought to be as low as possible. Current techniques were considered to fulfil these requirements inadequately (Erlanger and Blackman, 1910 ; Starzl, Gaertner, and Baker, 1955 ; Weirich, Paneth, Gott, and Lillehei, 1958 ; Watt and Pruitt, 1964).

The conduction system lies immediately under the endocardium. The first principle of our technique is based on this anatomical fact. Selective coagulation of a conduction bundle is possible by means of a diathermic probe (electrode) placed on the overlying endocardium. Such an electrode can be introduced into the heart through a minute incision. Thus a closed method, without the risks of open heart surgery, is feasible.

Nevertheless, intracardiac inspection could be useful to determine the exact endocardial area to be coagulated. It is undesirable, however, as it needs a cardiotomy and this results in a myocardial scar, which often conflicts with the scheduled experiment and adds to the operative risk. Therefore, anatomical and physiological principles were used to position the electrode properly without visual orientation.

These principles enabled us to produce nearly every type of block with minimal cardiac trauma. The mortality rate is hardly more than that of a thoracotomy and that of the resulting conduction disturbance. The varieties of heart block possible using this technique are demonstrated below by the description of two examples, i.e., the production of total heart block and of left bundle branch block. Details about general surgical care and anaesthesia in the animals used (dogs) are not given.
For experimental studies on the spread of myocardial activity in the auricles (where presumably specific conduction tissue is absent) with the application of the coagulation technique the reader is referred to former publications (Wieberdink, Meijler, and Durrer, 1961; Meijler, Wieberdink, and Durrer, 1962).

THE 'CLOSED' PRODUCTION OF TOTAL HEART BLOCK BY ELECTROCOAGULATION

A right-sided thoracotomy through the fourth or fifth intercostal space is used. The lung is retracted backwards. The pericardial sac is opened in front of the phrenic nerve and retracted with stay sutures. The easiest point at which to introduce the electrode lies in the lateral wall of the right auricle opposite (to the right of) the atrioventricular node. This point can be found, according to the size of the dog, 0.5 to $2 \mathrm{~cm}$. antero-cranially to the entrance of the inferior caval vein. Here, a purse-string suture (silk 30 ) is placed with a light tourniquet (for example, a piece of polyethylene tubing). Through an incision of 1 to $2 \mathrm{~mm}$. length the electrode is introduced (Fig. 1).

The electrode consists of a copper rod 10 to $15 \mathrm{~cm}$. long and 2 to $2.5 \mathrm{~mm}$. thick, covered by insulating polyethylene tubing. Its intracardial tip is bluntly rounded (Fig. 2a). The other end of the rod is connected to the diathermy apparatus. During thoracotomy this is pre-set in such a way that the electrode on the pectoralis muscles causes a visible area of coagulation of about $5 \mathrm{~mm}$. diameter if the current is closed for one second. A foot switch is used because this leaves the operator's hands free and enables the current to be switched on and off without movement of the electrode.

A rough determination of the area to be coagulated is made by the left index finger, pushed downward to the base of the aorta behind the right auricular appendage. The electrode, guided by the right hand, has to remain caudad, in medium-sized dogs some millimetres away from the tip of this finger. The rod is advanced into the heart perpendicularly to the operating table (the dog lying on its left side) until the 


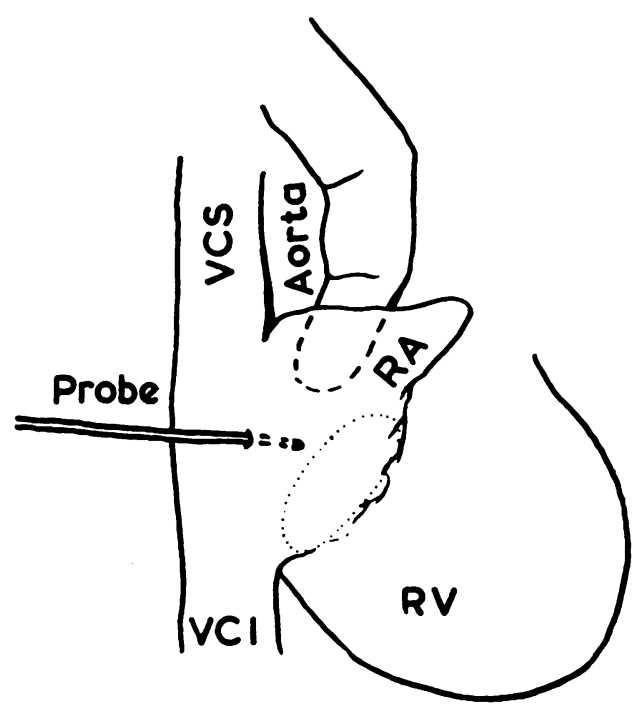

Total heart block

FIG. 1. Index finger palpates aortic root behind auricle. Dotted line: tricuspid orifice.

resistance of the interatrial septum is felt. If the electrode has been directed too far caudally, the movements of the septal leaflet of the tricuspid valve can be felt. Dorsally, the area where the bundle of His lies is limited by the line connecting the caval veins or even more anteriorly, since the atrioventricular conduction system begins ventral to the coronary sinus. Operating on big dogs, the beginner can facilitate his orientation by the introduction of the left index finger into the right auricle through a separate incision and purse-string suture; after some experience this manœuvre becomes unnecessary.

The detection of the exact point to be coagulated has been tested in several ways, including direct electrocardiographical leads from the electrode. None has proved simpler, quicker, and (after some experience) more effective than directly watching the heart. The critical phenomenon is delay of ventricular contraction as soon as the tip of the rod contacts the bundle of His. For this purpose gentle manipulation is required, because this enables repeated tests to be made: rough pressure on the bundle causes persisting disturbances that hamper renewed trials.

If pressure tests in one direction have repeatedly caused retardation of conduction, the rod is again advanced against the septum while the foot switch is pressed for one second. If contact has been made at the right point, total block follows immediately.

The rhythmic contractions of the auricles proceed unaltered, whilst a period of ventricular asystole begins. During this period the ventricles dilate; one gets the impression that they are 'pumped' (insufflated) by the auricles. Usually many seconds elapse before the ventricles start to contract in their own slow rhythm. By this time they have attained an alarming size and become cyanosed. Is this an imitation of the cardiac disturbances that occur duning an AdamsStokes attack?

In most cases the experienced 'blocker' will contact the bundle at the first attempt. The essential part of the operation will be completed in a few minutes, but the whole procedure takes about one hour. The less his experience, the more frequently will the surgeon have to reposition the probe because the first attempt has shown no evidence of success, there being incomplete or temporary interruption of the atrioventricular conduction. In this case, the following line of conduct is advised in order to avoid ultimate failure.

First, the tip of the electrode must be clean. As $\mathbb{D}$ every attempt at coagulation results in a deposit on the bare copper surface of the rod, isolation from the atrial wall will develop progressively unless this surface is mechanically cleaned after every electrical application. To omit this precaution renders precise orientation as well as circumscribed coagulation impossible. It will result in failure to produce a block and/or in damage to neighbouning structures (tricuspid insufficiency, septal defect, large thrombosed surfaces, etc.).

Secondly, a renewed attempt should not be made before the unsatisfactory result of the last is $\mathbb{D}$ stabilized. For several minutes after an unsuccessful coagulation rapid changes (progression as well as regression) in conduction may occur. When these have settled it is easier to judge renewed pressure tests and to find the correct point for final coagulation. The electrocardiogram is indispensable at this stage.

If the above steps are followed failure to achieve permanent total block will be rare. A safe policy in order to prevent late disappointments includes one

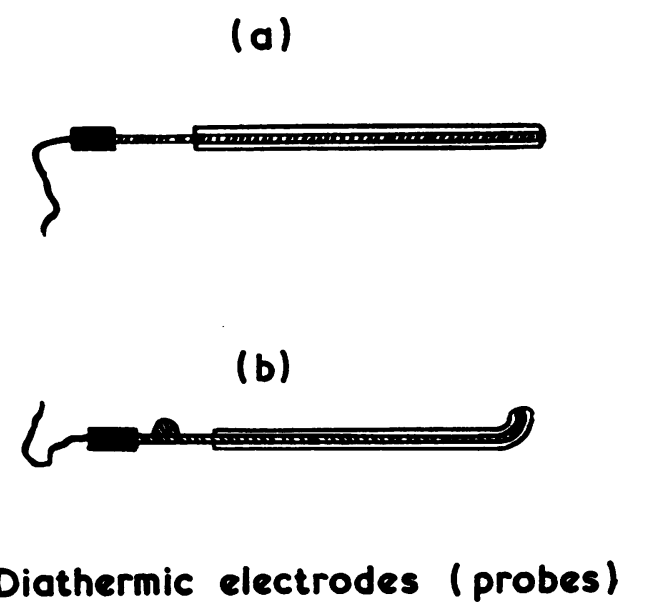

FIG. 2. Diathermic electrodes (probes). 
half hour of waiting after completion of the block. If no electrocardiographic signs of atrioventricular conduction recur during this period, it may be assumed that the block is stable.

The chances of survival of the dog can be improved by artificial pacemaking of the ventricles (Starzl and Gaertner, 1955), as acute total heart block may result in cardiac failure and death. Other post-operative risks are negligible, being in fact those of a thoracotomy, e.g., empyema and of lesions caused by imperfect coagulation technique (see above). Ventricular fibrillation during the operation is rare and ensues probably only if the coagulating electrode has touched the right ventricular wall or septum. For safety reasons a cardiac defibrillator should be at hand.

The operation is terminated by extraction of the electrode while the purse-string suture is tied. As the heart has become grossly dilated (if no pacemaker is used) the pericardium is not closed; with a few stitches its margins are sutured to the surface of the heart. The thorax is closed, using temporary watersealed drainage. No compensation for blood loss is required.

THE 'CLOSED' PRODUCTION OF LEFT BUNDLE BRANCH BLOCK BY ELECTROCOAGULATION A median sternotomy, although not tolerated well in the dog, gives the neeessary access to the heart. The lesion to the thoracic cage can be minimized by using an oscillating bone saw and by splitting only the caudal two-thirds of the sternum. As both parts are flexible, a Finochietto spreader will make the desired manipulation at the cardiac apex and digital palpation at the aortic root possible.

The pericardial sac is opened in the median line. Its margins are raised by stay sutures. A purse-string suture (silk 30) with a light tourniquet (polyethylene tubing) is inserted into the apex of the left ventricle. This allows the introduction of the electrode with haemostasis and minimal impairment of cardiac function.

The electrode used in this case has the same construction as that of the one described for the coagulation of the atrioventricular bundle. Its shape, however, is adapted to the demands of orientation and coagulation in the left ventricle. The intracardiac tip has a short $90^{\circ}$ curve, while a wing at the other end of the rod enables the operator to control the direction of the tip during intracardiac manipulation (Fig. 2b).

In spite of this curve the rod can easily be introduced through a small stab-wound in the apex. It is advanced (its tip pointing to the front) through the left ventricle and the aortic valve until the tip can be palpated with the left index finger, resting on the anterior surface of the aortic root. This tip has to be brought into contact with the endothelium overlying the left bundle branches.

In contrast to the procedure described for the production of total block, orientation cannot be based on function tests because the left bundle divides early in several branches. The physiological response to pressure with the tip of the rod will therefore not give conclusive information. Anatomically, a line of coagulation has to be produced, crossing the branches of the left bundle just caudal to the point where they reach the left subendocardial layer of the interventricular septum. Depending on the size of the dog this line runs 5 to $10 \mathrm{~mm}$. below the aortic valve, parallel to a part of the aertic ring. It will cross all left bundle branches if a $135^{\circ}$ sector of the ring is circumscribed, from $45^{\circ}$ right-dorsal to frontal.

In order to bring the tip of the electrode to the desired level, the rod is slowly retracted with the right hand until the sensation that its curved tip hooks on one of the cusps of the aortic valve is registered. Some gentle manipulation will let this cusp free itself, making possible further extraction of the rod; after 5 to $10 \mathrm{~mm}$. it is stopped (Fig. 3).

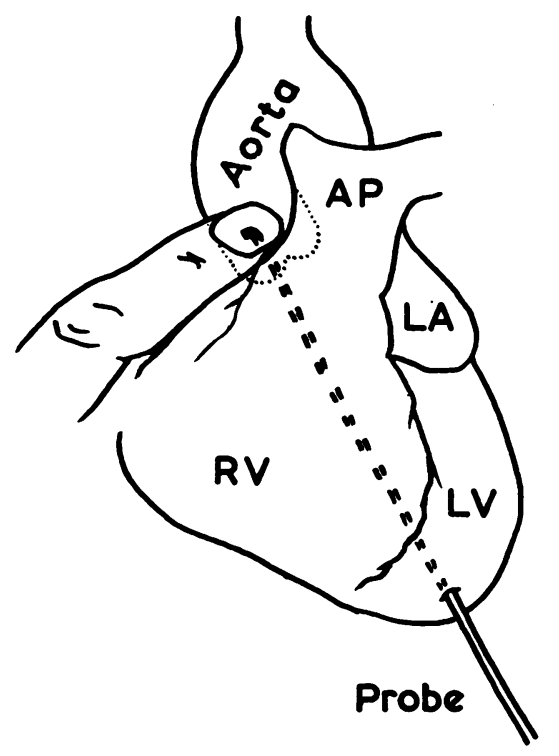

\section{Left bundle branch block}

FIG. 3. Index finger palpates curved tip of probe in base of aorta. Dotted line: aortic valve.

During coagulation the rod describes a rotating movement over the above-mentioned $135^{\circ}$ from frontal to right-dorsal, or in the opposite direction. The tip of the electrode must be pressed against the endocardium in order to make electrical contact, though not too firmly because this would prevent it from gliding over the uneven endocardial surface. The foot switch is pressed until the rotation is completed, which takes about 5 seconds.

This coagulation will often lead to ventricular fibrillation. Immediate defibrillation is always effective. Therefore induced fibrillation was examined as a 
means of facilitating orientation and coagulation, making work on a 'quiet heart' possible. No advantages could be demonstrated from this technical variant.

The complete interruption of all left bundle branches is much more difficult to achieve than total heart block. In about half of our cases partial conduction remained: in spite of patient additional attempts to coagulate all branches the electrocardiogram failed to show the desired effect. Knowing no other technique of producing permanent left bundle branch block without serious concomitant damage, e.g., ventriculotomy, to the heart, this result was considered acceptable (Philippi, A. W., in preparation).

To reach this result it proved useful to check the effect of the coagulation in cases of failure at necropsy. The left bundle branches can reasonably be recognized on macroscopic inspection of the interior of the heart, while the track of the electrode is also visible. Undesirable lesions, such as aortic insufficiency, will be obvious and contribute to the gradual improvement in blind orientation as well as in coagulation technique.

Termination of the operation requires closure of the apical incision, some stitches to approximate the pericardial margins, temporary water-sealed drainage of the thorax, reconstruction of the sternum, and closure of the wound.

\section{SUMMARY}

A technique for the experimental production of artificial heart block (total atrioventricular as well as different kinds of bundle branch block) is described.

Our requirements have been that $(a)$ the block must be permanent, and $(b)$ the lesion to the heart, necessary for the production of the block must be negligible.

The technical principles involved are $(a)$ theo block is created by electrocoagulation, using an ${ }^{2}$ electrode brought into selective contact with theendothelium overlying the part of the conduction: system to be destroyed; $(b)$ orientation and $\vec{\omega}$ coagulation with this electrode are done without interruption of the circulation.

The technique can be described as a closedi method which allows selective coagulation of $\overrightarrow{\mathrm{r}}$ parts of the conduction system.

The possibilities of this technique are demonstrated by a description of operations for the pro-은 duction of total heart block and left bundle branchblock. From these examples the application of $\mathbb{\Phi}^{\infty}$ the technique for the production of other types? of conduction disturbances can be worked out.

\section{REFERENCES}

Erlanger, J., and Blackman, J. R. (1910). Further studies in the physi-o ology of heart-block in mammals. Chronic auriculo-ventricular heart-block in the dog. Heart, 1, 177.

Meijler, F. L., Wieberdink, J., and Durrer, D. (1962). L'importance de la position des électrodes stimulatrices au cœurs du traitement d'un bloc auriculo-ventriculaire post-opératif total. Arch. Mal $\bar{\partial}$ Cour, 55, 690.

Starzl, T. E., and Gaertner, R. A. (1955). Chronic heart block in dogs. A method for producing experimental heart failure Circulation, 12, 259 .

dogs. Ibid., 12, 82 .

Watt, T. B., and Pruitt, R. D. (1964). Cocaine-induced incomplets bundle branch block in dogs. Circulat. Res., 15, 234.

Weirich, W. L. Paneth, M. Gott, V. L., and Lillehei, C. W. (1958)C Control of complete heart block by use of an artificial pacemaker and a myocardial electrode. Ibid., 6, 410.

Wieberdink, J., Meijler, F. L., and Durrer, D. (1961). Artificial pace. making in experimental and clinical heart block. Minerva Cardio angiol. europ., 9, 310 . 